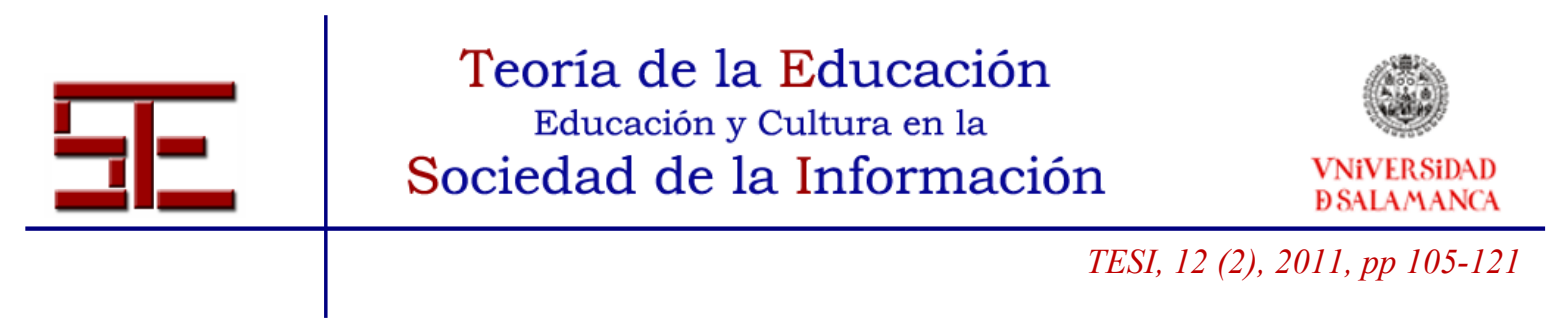

\title{
INDAGANDO EN LA RELEVANCIA DE INTERNET EN EL ACCESO, USO Y DESEOS DE LAS TIC POR PARTE DE LAS MUJERES EN LAS TIC
}

Resumen: A partir de los años 90 las teorías feministas de la tecnología experimentan un optimismo renovado con la aparición de las TIC y el desarrollo de la tercera ola feminista. Sin embargo, la investigación en género y tecnología se ha centrado en estudiar la exclusión de las mujeres de las TIC e, inversamente, las experiencias de las mujeres y sus estrategias para incluirse en las TIC han recibido menos atención. Motivadas por nuestras experiencias tecnoactivistas y la observación de una remarcable presencia de mujeres en ellas, con Donestech, iniciamos un proyecto de investigación sobre los accesos, usos y deseos de las mujeres en las TIC. A través de una investigación ciberfeminista recogimos, analizamos y visibilizamos las experiencias y opiniones de las mujeres en las TIC. Nuestros resultados sugieren que las trayectorias de inclusión y acceso de las mujeres en las TIC son diversas, así como heterogéneos son los usos que hacen de ellas, destacando el rol jugado por Internet en todo ello. Además mostramos que las mujeres en las TIC son curiosas y entusiastas, que quieren empoderarse y compartir el conocimiento. Finalmente, les disgusta y desconfían de las relaciones de poder existentes en los mundos tecnológicos, manteniendo una actitud crítica y creativa hacia las TIC.

Palabras clave: Tecnología; género; aprendizaje; usos; deseos; e-inclusión; Internet; investigación activista; ciberfeminismo.

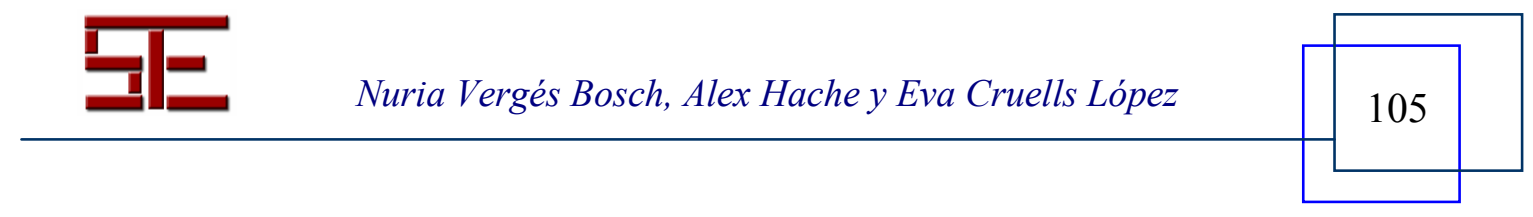




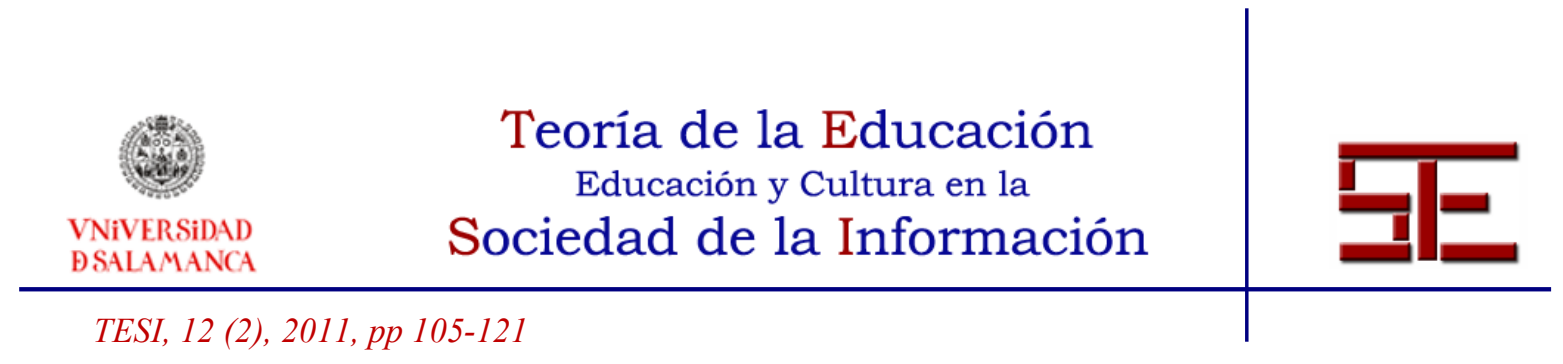

\title{
DIGGING IN THE SIGNIFICANCE OF THE INTERNET FOR THE ICT ACCESS, USES AND DESIRES OF WOMEN IN ICT
}

\begin{abstract}
Since the 90's the feminist theories of technology have experienced a renewed optimism with the rise of ICT and the development of the third-wave feminisms. However, Research on gender and technology still focuses on studying the exclusion of women from ICT. Conversely, women's experiences and their strategies leading to their inclusion in ICT has received less attention. Motivated by our previous experience in technoactivism and the observation of a remarkable presence of women, within the Donestech Collective, we initiated a research project on the access, uses and desires of women who were involved in technology. Following a cyberfeminist activist research approach we collected, analysed and made visible the experiences and opinions of women in ICT. Our findings suggest a great diversity of women trajectories to ICT as well as heterogeneous technological practices among them where Internet plays a significant role. Moreover, our results show that women in ICT are curious and enthusiastic, want to be empowered, are willing to share the knowledge gained with others, distrust and dislike power relations existing in the technological environments and try to maintain a critical and creative attitude towards technology.
\end{abstract}

Keywords:Technology; gender; learning; uses; desires; e-Inclusion; Internet; activist research; cyberfeminism.

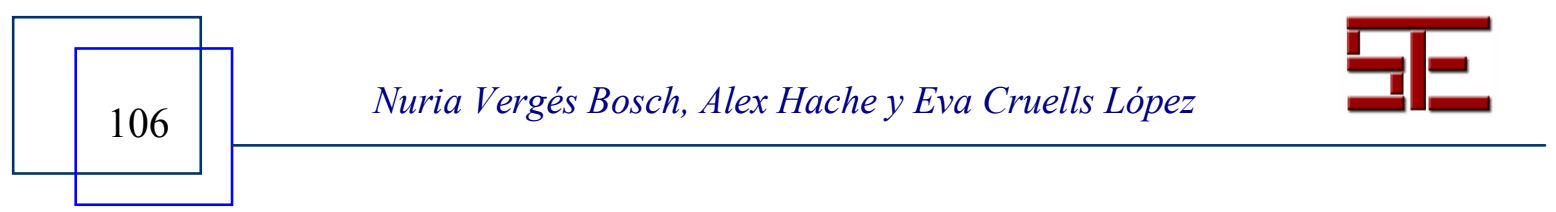




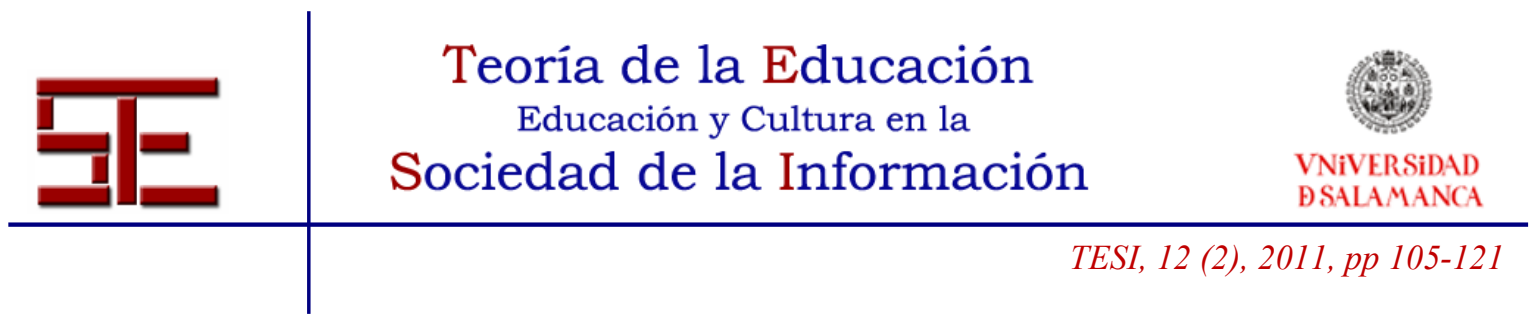

\section{INDAGANDO EN LA RELEVANCIA DE INTERNET EN EL ACCESO, USO Y DESEOS DE LAS TIC POR PARTE DE LAS MUJERES EN LAS TIC}

Fecha de recepción: 11/05/2011; fecha de aceptación: 12/06/2011; fecha de publicación: 27/07/2011

Núria Vergés Bosch

nverges@uoc.edu

Grupo de investigación Copolis. UB; Colectivo Donestech.

Alex Hache.

alex.hache@gmail.com

Colectivo Donestech

Eva Cruells Lopez.

cruels@,gmail.com

Colectivo Donestech.

\section{INTRODUCCIÓN}

A partir de los años 90, las teorías feministas de la tecnología experimentan un optimismo renovado por el auge de las TIC y el desarrollo de la tercera ola feminista. Partiendo del potencial de las nuevas tecnologías, y en particular de Internet, para la transformación de la sociedad y de las mujeres, el feminismo se recarga a través de la renovación tecnológica, a la práctica y de forma teórica. Estas feministas invitan a la participación de las mujeres en las TIC animadas por las oportunidades que conllevan para las transformaciones de género (Haraway, 1991; Plant, 1997). Actualmente, las teóricas feministas de la tecnología se muestran menos optimistas y más conscientes del hecho de que la tecnología también está generizada de una forma en que el género y la tecnología devienen mutuamente constitutivos (Wajcman, 2006; Sveningsen y Sunden, 2007; Landstrom, 2007). De esta manera, mientras critican las desigualdades de género enfatizan las posibilidades de participación de las mujeres en las TIC para sobrepasar estas disparidades y facilitar la transformación de la sociedad. Este potencial de transformación radica en las nuevas oportunidades de apropiación de las mismas TIC por parte de las mujeres, ya que las TIC se desarrollan de forma horizontal y facilitan relaciones horizontales (Hawthorne y Klein, 1999; Núñez y García, 2009). También se considera que radica en las posibilidades de creación y ocupación de nuevos espacios como el ciberespacio (Flanagan y Booth, 2002; Sveningsen y Sunden, 2007) y, sobre

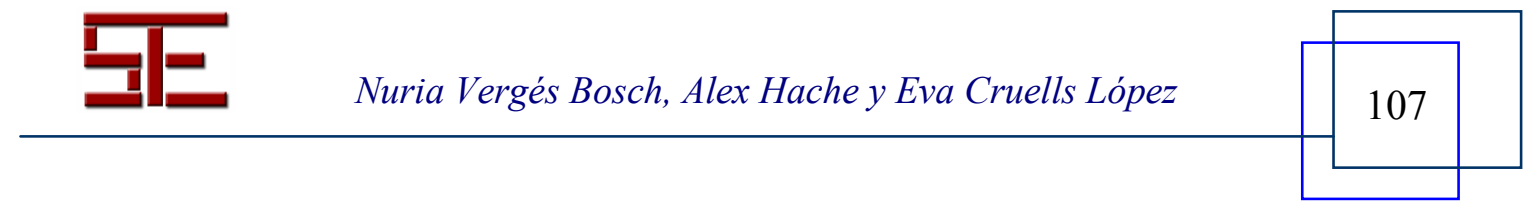




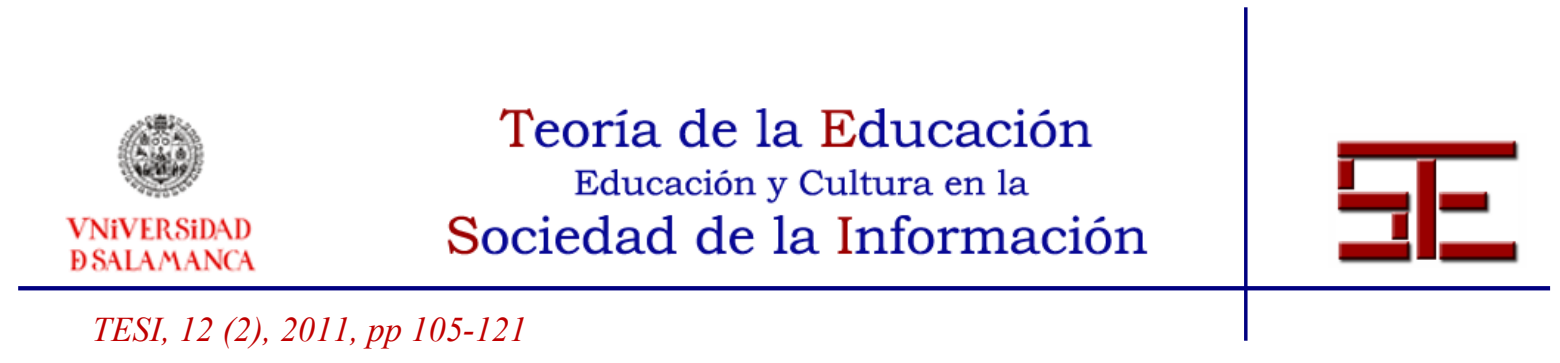

todo, en el poder reconstruir y deconstruir categorías predeterminadas, así como los mismos binarismos que se aplican al género y la misma interpretación que se hace del concepto de tecnología (Haraway, 1991; Wajcman, 2004; Landstrom, 2007).

A pesar de estos avances teóricos, la investigación sobre las mujeres en las TIC se sigue centrando en explicar los mecanismos de exclusión de las mujeres (Cohoon y Aspray, 2006; Castaño, 2008). Así, las experiencias y estrategias de participación de las mujeres en las TIC han recibido menos atención. Como ya han apuntado diversas autorías (Sorensen, 2002; Lagesen, 2007; Faulkner y Lie, 2007), es necesario entender más profundamente los mecanismos actuales de inclusión TIC para las mujeres.

Esta necesidad llevó a nuestro colectivo de mujeres y tecnologías, Donestech (Dones es la palabra en catalán para mujeres y tech se refiere a tecnología), a específicamente interesarse en los procesos de inclusión de las mujeres en las TIC y tratar de conocer y explicar sus trayectorias de acceso a las TIC, así como sus usos y deseos como usuarias avanzadas y desarrolladoras TIC.

Hasta el momento, la investigación feminista de la tecnología centrada en los procesos de inclusión ha identificado ciertas motivaciones que llevan a las mujeres a interesarse por las TIC. De acuerdo con lo apuntado por Sorensen (2002), estas motivaciones se resumen, por un lado, en las de duty, que expresan una motivación utilitaria y de herramienta, $\mathrm{y}$, por otro lado, en las de love, que expresan una motivación entusiasta, pasional o de interés por las mismas TIC. Curiosamente, el utilitarismo se ha atribuido tradicionalmente a la mujer, mientras que el entusiasmo se ha atribuido a los hombres. De esta forma, la distribución motivacional de género niega e invisibiliza las experiencias de acceso de las mujeres en las TIC motivadas por el entusiasmo (como crítica Sorensen, 2002; Lagesen, 2008).

Aunque todavía escasa, la literatura previa relacionada con el género y la e-inclusión ha identificado una serie de factores facilitadores de la entrada e inmersión de las mujeres en las TIC (Margolis y Fisher, 2001; Sorensen, 2002; Lagesen, 2007, 2008; Faulkner y Lie, 2007). Brevemente y en primer lugar, se consideran los factores que identifican la importancia del contexto, potencialmente (o percibido) como amigable para las mujeres. Este sería el caso de situaciones en que se contestan los insultos sexistas o se respetan las normas laborales en cuanto a la maternidad. En relación a ello, y en segundo lugar, también hace falta que existan herramientas, espacios y contenidos de interés o útiles para las mujeres, como, por ejemplo, contenidos de aprendizaje relacionados con

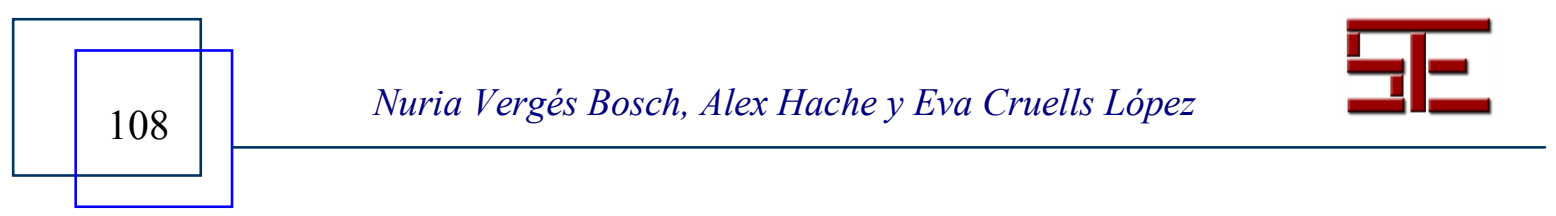




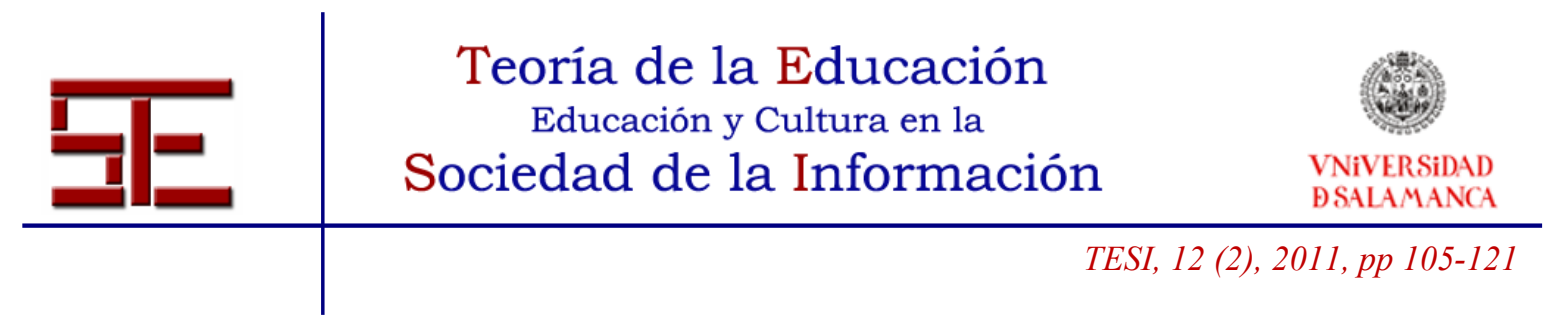

aspectos de salud que suelen afectar a las mujeres. En tercer lugar, como en cualquier otro tipo de aprendizaje, es necesario que la información sea disponible así como que exista oferta de recursos de aprendizaje. De este modo el hecho de que la información circule libremente o de que existan foros para compartir dudas sería un facilitador en este sentido. En cuarto lugar y en relación a ello, las infraestructuras y los equipos o dispositivos de acceso a las TIC (ordenadores, bibliotecas de libre acceso a Internet, banda ancha $u$ otros...) siguen siendo relevantes para facilitar la e-inclusión y por ello sigue siendo relevante la existencia de políticas públicas al respecto que, además del género, tengan en cuenta otras cuestiones socioeconómicas. En quinto lugar, la existencia de role models o de otro tipo de mecanismos como el mentoring, tutoring, coaching $\mathrm{u}$ otros mecanismos de apoyo a la inclusión que se puede facilitar tanto visibilizando el rol histórico de las mujeres en el desarrollo de las tecnologías como facilitando la creación de redes o grupos de afinidad para compartir el conocimiento. Finalmente, la percepción de las mujeres respecto a las expectativas de empleo u otro tipo de beneficios sigue siendo relevante para la inclusión. Así, tener en cuenta estos elementos laborales relacionados con tiempos, estabilidad y retribuciones constituye un prerrequisito para facilitar el acceso e inclusión de las mujeres en las TIC, en la medida que ello las empodere y facilite su inmersión más profunda hasta participar en el diseño $\mathrm{y}$ desarrollo de las TIC de una forma activa, crítica y desde una perspectiva emancipadora.

\section{CIBERFEMINISMO DE INVESTIGACIÓN}

Motivadas por nuestras experiencias tecnoactivistas previas y la observación de una remarcable presencia de mujeres en ellas, en el 2006 y en el marco del colectivo Donestech, iniciamos un proceso de investigación activista para conocer por qué y cómo las mujeres habían accedido a las tecnologías, cómo las usaban y qué deseos de mejora expresaban. Nuestro objetivo consistía en ofrecer una plataforma de expresión y análisis de sus experiencias y opiniones, mientras intentábamos descifrar el código LeLa, sobre las relaciones de las mujeres y las tecnologías. En vez de insistir en la cuestión de por qué las mujeres no estaban en las tecnologías, nos preguntábamos por qué las mujeres habían accedido a ellas, qué las había motivado, qué lo había facilitado, cómo eran sus trayectorias TIC, qué herramientas usaban y para hacer qué y qué tácticas o estrategias seguían para superar cualquier limitación encontrada, pero también para seguir construyendo su relación con la tecnología. Todo ello para visibilizar las especificidades de las mujeres en las TIC, a la vez que, nosotras mismas, continuábamos creando redes e incorporando las tecnologías en nuestra investigación y en nuestras prácticas tecnoactivistas. Como ya apuntamos en nuestro manifiesto colgado en la web,

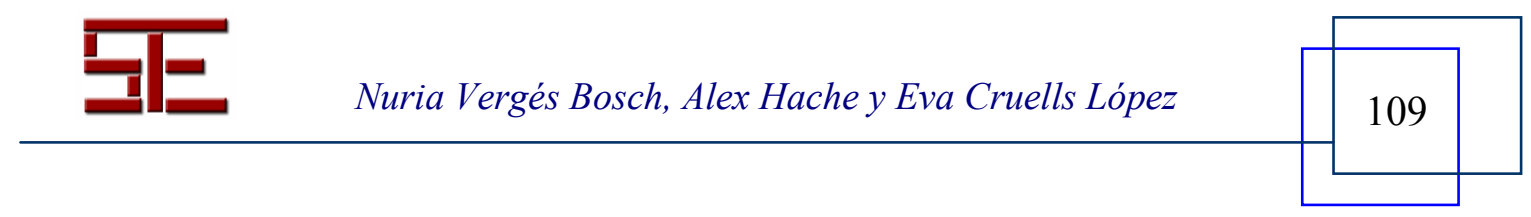




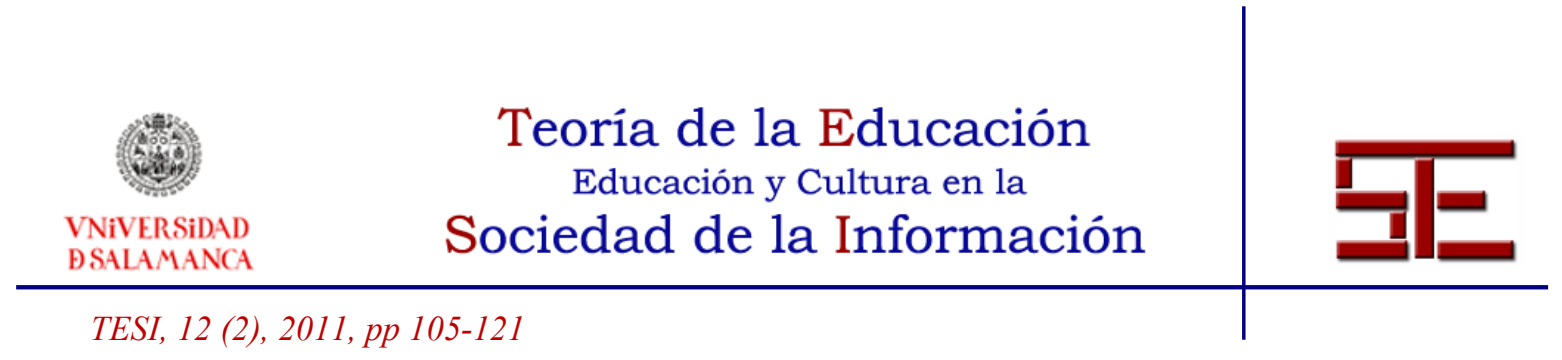

nos hemos ido enredando más y más y más... de manera que el proyecto Código LeLa se ha convertido en un teraproyecto de lo que relaciona los géneros y las tecnologías.

Nuestra investigacción ciberfeminista se basa en un uso y práctica intensiva con las TIC que intenta, a través de la producción de conocimiento crítico, la acción creativa o el activismo social y artístico generar transformaciones sociales y de género desde una perspectiva dinámica, fluida, heterogénea y preformativa (Vergés et al. 2010). De acuerdo con ello, creamos un portal web (www.donestech.net), recogimos información sobre mujeres y tecnologías, generamos y participamos en talleres, encuentros y eventos, realizamos creaciones audiovisuales y artísticas y nos focalizamos en mapear, identificar, compilar, analizar y comunicar las experiencias y opiniones de mujeres en las TIC que quisieran compartidas. Aunque inicialmente estábamos basadas físicamente en Barcelona, nuestra presencia en el ciberespacio fue creciendo y las participantes en Donestech empezaron a convertirse en geográficamente nómadas. Por ello, tendimos a buscar y diseminar la información en diferentes lenguas como el catalán, el español, el inglés o el francés, sobre todo, a través de Internet.

Específicamente y de acuerdo con lo que presentamos en este artículo, hicimos una llamada a través de Internet para la respuesta de nuestro cuestionario semi-cualitativo contestable on-line. Así, simulando la técnica de bola de nieve a nivel virtual, enviamos la propuesta a listas de correo especializadas y a diferentes colectivos, asociaciones o instituciones de mujeres o de tecnologías. Paralelamente, entre el 2007 y 2008, llevamos a cabo múltiples entrevistas semi-estructuradas en profundidad de forma presencial, además de distintos grupos de discusión. Aunque empezamos nuestra investigación en el contexto catalán, pronto la extendimos al contexto español con algunas incursiones internacionales. Las informaciones presentadas en este artículo, pues, se basan en 302 cuestionarios on-line, 60 entrevistas en profundidad a mujeres en las TIC que se contactaron a través de entidades y eventos tecnológicos o de mujeres, además de siguiendo la bola de nieve. Finalmente, aunque en menor medida, se basan en los 3 grupos de discusión realizados en distintas Comunidades Autónomas, Andalucía, Cataluña y Madrid.

Para su recogida, análisis y divulgación llevamos a cabo una triangulación metodológica con un enfoque pluridisciplinar, así como un uso intenso de las tecnologías acercándonos a las prácticas ciberfeministas. De este modo, incorporamos disciplinas tan diversas como la sociología, la informática, la comunicación audiovisual o las bellas artes y utilizamos métodos estadísticos, métodos de visualización

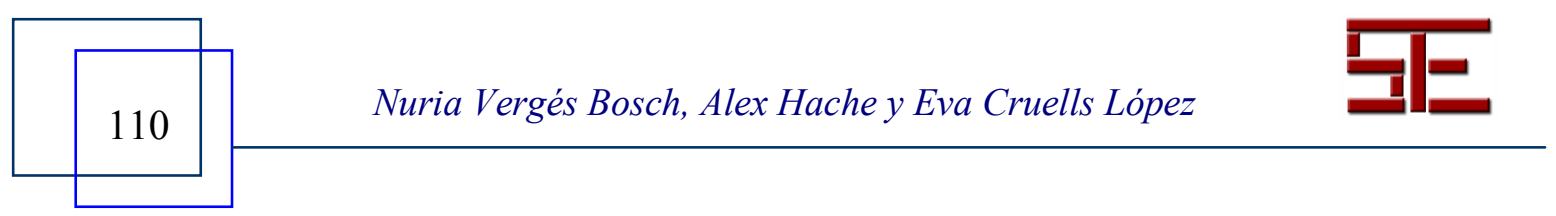




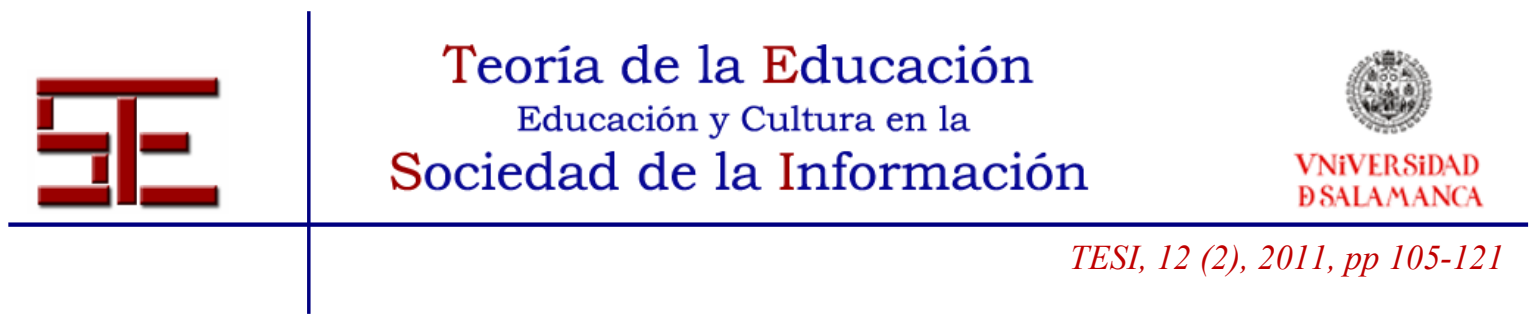

interactiva, multimedia y audiovisual, así como, realizamos un análisis de contenido temático de las narrativas compartidas.

\section{ACCESOS, USOS Y DESEOS DE LAS MUJERES EN LAS TIC. LA RELEVANCIA DE INTERNET}

En nuestra investigación nos propusimos no partir de una definición acotada o predeterminada de mujer y tecnología, a fin de no caer en un universo predefinido y específico de mujeres tecnólogas. Las mujeres participantes en nuestra investigación resultaron ser mujeres diversas y usuarias avanzadas de las TIC, pero también creadoras y desarrolladoras de las TIC de una forma muy heterogénea.

Sus trayectorias TIC también resultaron ser diversas y podrían agruparse en trayectorias lineares, interrumpidas y nómadas. Las trayectorias lineares son aquellas donde a partir de un primer contacto con las TIC se ha continuado directamente en su aprendizaje y práctica de forma constante y lineal, por ejemplo, estudiando ciencias puras, estudiando posteriormente ingeniería informática o telecomunicaciones, para después trabajar de programadora y más adelante de analista o jefa de proyectos TIC. Este sería el caso de algunas de las mujeres ingenieras, aunque no todas. En este tipo de trayectorias predomina el aprendizaje reglado combinado, sobre todo, en etapas posteriores del curso de vida, con el aprendizaje informal. Las trayectorias interrumpidas serían buena parte del resto si consideráramos sólo la infancia y adolescencia, pues como veremos se observa un importante vacío desde el primer contacto hasta el inicio que suele ser en la universidad o ya en el trabajo. De este modo las trayectorias interrumpidas serian aquellas que más allá de este vacío inicial presentan un distanciamiento brusco o profundo con las TIC para un período determinado de su vida. Finalmente, numéricamente y cualitativamente destacan las trayectorias nómadas. Este tipo de trayectoria implica una línea menos profunda y mucho más curva que para la trayectoria lineal y un distanciamiento menos profundo o brusco respecto a las trayectorias interrumpidas. Por ejemplo, este sería el caso de mujeres ingenieras que se han desplazado a otros sectores de actividad a la vez que han disminuido la intensidad de su dedicación TIC o sería el caso de artistas que en sus estudios de arte han tendido a escoger asignaturas o itinerarios relacionados con la imagen digital, interactivos $o$ similares. Para este tipo de trayectoria las fórmulas de aprendizaje tienden a ser no formales combinadas con las informales o con la nueva formación transdisciplinar reglada $\mathrm{y}$, a su vez, los usos tecnológicos mucho más heterogéneos que en las trayectorias anteriores.

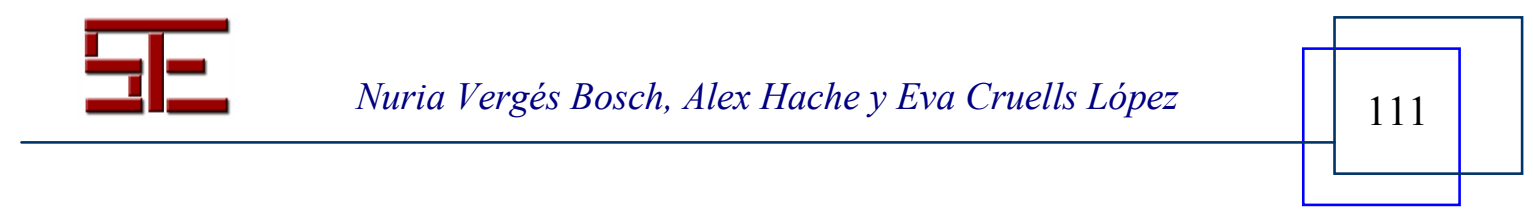




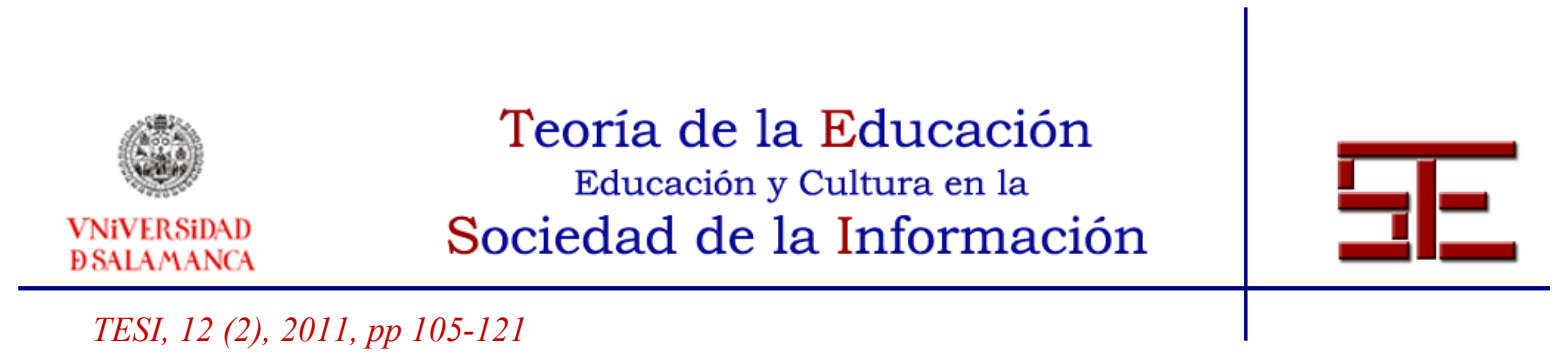

Pese a la diversidad de perfiles y trayectorias observadas, el perfil más común de las mujeres tecnólogas que componen nuestra población lo constituyen mujeres relativamente jóvenes, de grandes ciudades, con estudios universitarios, con trabajos remunerados y una posición social media-alta, pero económicamente media-baja. Sobre todo son solteras, tienen pareja, tiempo libre y normalmente no tienen personas a su cargo. Esto implica que en la elaboración de estrategias de inclusión además del género cabe tener en cuenta las desigualdades por lugar de origen, clase y edad, pues las mujeres mayores, de zonas rurales y sin estudios o con pocos recursos económicos pueden estar doblemente excluidas de lo tecnológico.

En las siguientes secciones expondremos pues, en primer lugar, sus experiencias de acceso y aprendizaje. En segundo lugar, sus experiencias de uso y práctica tecnológica señalando algunos aspectos de sus condiciones. Finalmente, expondremos sus críticas y deseos expresados en relación a las TIC.

\subsection{Experiencias de acceso y aprendizaje...}

Nuestra metodología nos permite recorrer las trayectorias de acceso e inmersión en los conocimientos y entornos tecnológicos de las mujeres desde su primer contacto con las tecnologías hasta sus motivaciones, facilitadores y formas de aprendizaje posteriores.

Cuando les preguntamos sobre su primera experiencia buscábamos provocar un ejercicio memorístico que nos condujera a su toma de contacto o a su relectura posterior de ello, que, a la vez nos condujo, a nosotras mismas, a operar un proceso similar. Su primera vez se presenta muy variada, en casa, en la escuela y/o en el trabajo, desde usar artefactos domésticos hasta ordenadores, desde jugar a videojuegos hasta utilizar procesadores de texto o el correo electrónico. Acompañadas por un familiar como la tía, el padre, la hermana o apoyadas por la maestra, los compañeros de clase o algún amigo. Resulta inquietante el vacío temporal que presentan muchas de sus experiencias en el tiempo. Después de este primer contacto, muchas no son animadas, empujadas o alentadas a continuar en lo tecnológico y así, lo que señalan como su momento de verdadero acceso a las tecnologías se sitúa en un momento bastante posterior, como su entrada en la Universidad o el mundo laboral, como muestra una de las experiencias recogidas de una mujer que se dedica a la administración de sistemas informáticos: "Desde los tres años en casa ha habido ordenadores, pero no fue hasta 1998 que no empecé a meterme a fondo con la informática".

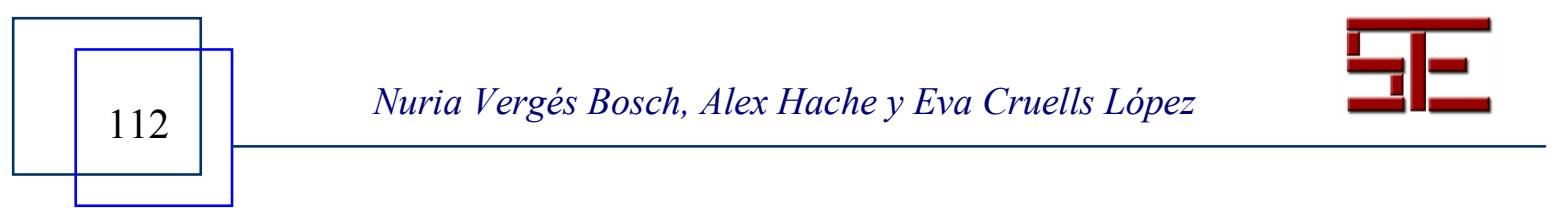




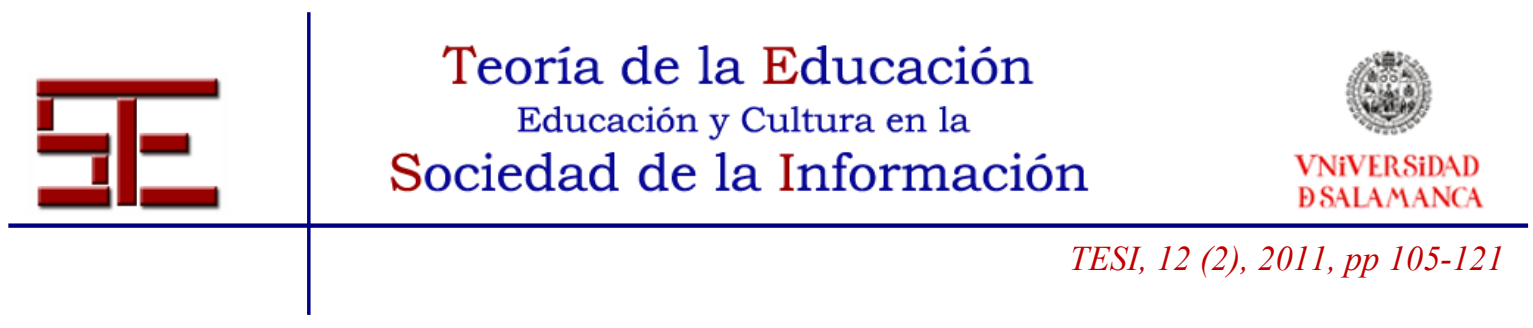

Así, aunque existe un vacío temporal entre su primer contacto y su inmersión tecnológica, sus primeras experiencias se relacionan, por orden de relevancia, con experiencias formativas; con aparatos varios; con Internet; la realización de tareas con programas específicos; y finalmente con los juegos, además de otras experiencias con la programación o el activismo. En relación a ello el rol de Internet es significativo, pues la existencia de Internet y sus posibilidades de búsqueda de información han sido el primer contacto para muchas mujeres, así como crear una cuenta de e-mail, utilizarla y comunicarse por Internet. Así lo expresó una diplomada en sistemas de Gestión del Bienestar: "Mi primer acceso a Internet y la creación de mi primera cuenta de correo electrónico".

En cuanto a las motivaciones de acceso sus experiencias muestran una gran variabilidad de motivos que a menudo se superponen. Sin embargo, en relación a lo apuntado por la literatura anteriormente, destacan las motivaciones entusiastas como el deseo de explorar, la curiosidad o la atracción al mismo nivel que aquellas motivaciones más relacionadas con finalidades utilitaristas para su educación y acceso a una profesión. Así expresa sus motivaciones entusiastas una ingeniera multimedia curadora de arte multimedia: "Estaba haciendo física y un amigo me enseñó un folleto de Ingeniería multimedia y yo dije, iyo quiero hacer flipes de estos! Compaginé durante un año física e ingeniería multimedia, para, finalmente, acabar multimedia”.

Los factores facilitadores de acceso también se muestran variados y mucho más superpuestos, de modo que difícilmente encontramos un solo factor de forma aislada. Por orden de relevancia se citan la posibilidad formativa; las posibilidades $\mathrm{o}$ necesidades de desarrollo profesional; la capacidad económica y la posesión de equipos; Internet y sus posibilidades comunicativas; la ayuda, apoyo o colaboración del entorno afectivo y social; el contexto actual marcado por la sociedad de la información; y otros factores como la posibilidad de destinarle tiempo o acceder a partir de prácticas activistas. Así pues, el hecho de tener acceso a Internet y a sus posibilidades se presenta también como un factor facilitador clave de acceso de las mujeres a las tecnologías como muestra el testimonio de esta periodista y realizadora audiovisual: "El hecho de tener un ordenador e Internet en casa desde muy pequeña. Y el hecho de estudiar Comunicación y necesitar constantemente de los ordenadores, documentación, edición de vídeos, maquetar etc".

Finalmente, las tecnólogas acceden al aprendizaje tecnológico a través de diferentes vías activadas de forma simultánea en las que sobre todo predomina la vía autodidacta e

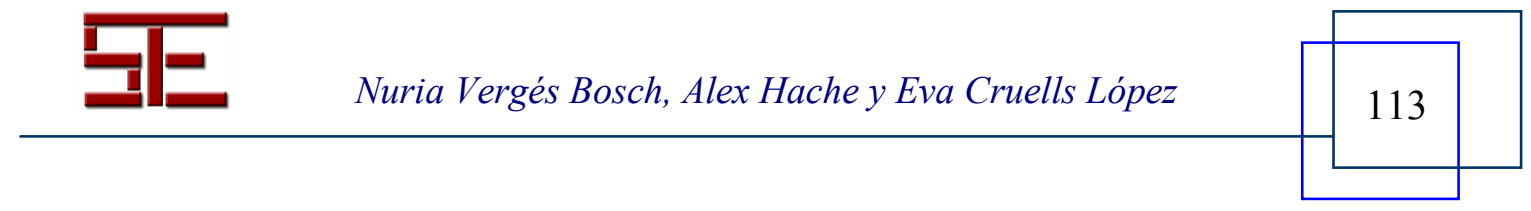




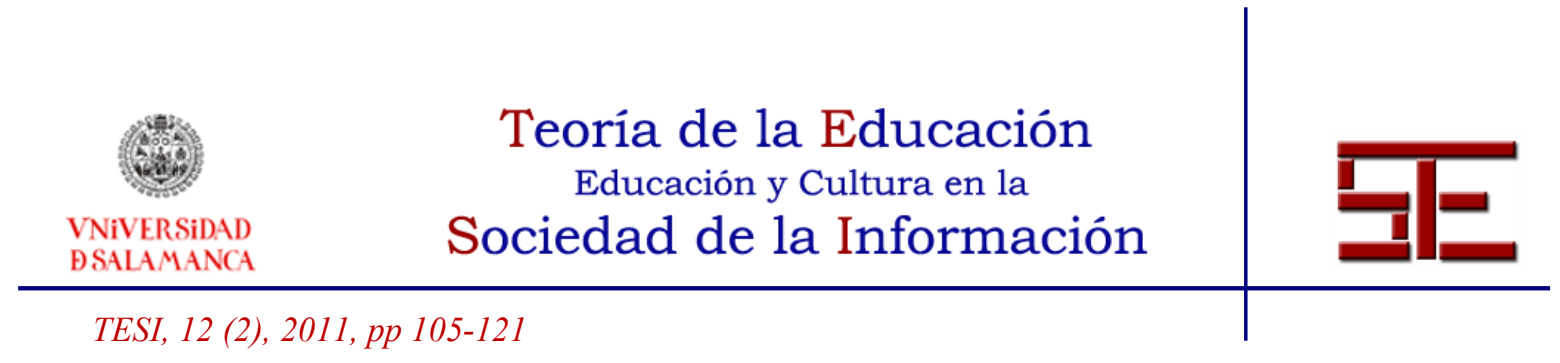

informal, aunque combinada con la formación no formal y con la reglada. Si atendemos a los estudios reglados cursados casi la mitad de las participantes no tiene estudios científico-técnicos, sino más bien propios de las ciencias sociales o las humanidades. Además la variabilidad disciplinar entre las mujeres de perfil científico-técnico es igualmente acusada. También como forma de aprendizaje no formal en colectivo asisten a cursos, talleres o jornadas con finalidades formativas respecto a un programa, aplicación o lenguaje específico que tienen intención de aprender. Entre las vías de aprendizaje y capacitación tecnológica experimentadas por las mujeres tecnólogas cabe destacar la importancia de las vías informales. La adquisición de conocimientos a través de estas se presenta con diferentes formas que se suceden e intercalan en el tiempo. La vía más autodidacta y solitaria se realiza a través del aprendizaje prueba-error con un equipo, programa o lenguaje de programación específico, utilizando manuales o información compartida en Internet a través de buscadores o páginas web especializadas. Otras veces el auto-aprendizaje se realiza de forma colectiva a través de contactos y/o amistades, redes, comunidades o foros, además de la participación en encuentros o eventos especializados como linux intall parties o hackmeetings. En relación a ello y de forma lógica, las mujeres con estudios técnicos realizan menos cursos específicos para la formación tecnológica, aun así, la presencia de las vías de aprendizaje informales, sobre todo para seguir formándose, son igualmente relevantes. Así lo muestra la experiencia de una mujer ingeniera en telecomunicaciones que se dedica a la ingeniería musical: "En realidad en los estudios de telecomunicaciones sólo hay una asignatura de programación. Yo no tuve más formación que esa. En realidad soy autodidacta, aprender programando. Al principio con tutoriales, un poco así. He aprendido sola más o menos. Yo soy bastante metódica, coger un manual e ir haciéndolo, así es como aprendí, y luego preguntando también. Y luego tengo mucho la filosofía de copiar y pegar, mente de copiar, de imitar, como la música, leo mucho, mirar mucho código y coger ideas".

La preponderancia del aprendizaje informal abre nuevas y diversas posibilidades de aprendizaje tecnológico. Por un lado, implica las posibilidades de acceso al conocimiento avanzado de la tecnología sin haber seguido una trayectoria linear, de modo que permite la entrada a partir de otras disciplinas al igual que el retorno después de la maternidad. Por el otro, se constituye como una característica de las mismas TIC, sobre todo de las tecnologías libres, que las hace especialmente atractivas para la entrada, pues, en cierta medida, no existen barreras formales de acceso y, a su vez, necesarias para el continuo reciclaje que el conocimiento y la práctica avanzada tecnológica requieren.

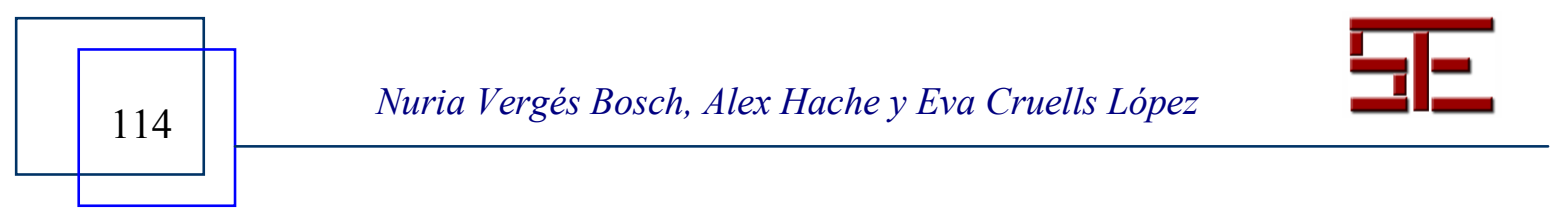




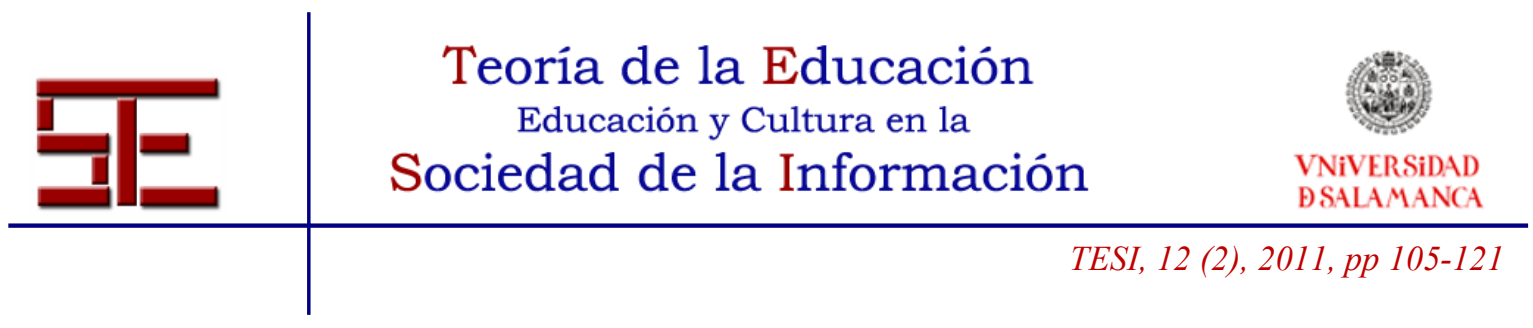

Ahora bien, a la vez, conlleva las dificultades y desventajas del aprendizaje no reglado. La formación informal requiere un importante grado de motivación, formación previa y de tiempo disponible para la búsqueda y experimentación. Además, el elevado grado de informalidad dificulta el establecimiento de una base común e igualitaria e incrementa las dificultades para la evaluación y reconocimiento de las capacidades adquiridas.

\subsection{Experiencias de uso y sus condiciones...}

Como en el análisis de las trayectorias de acceso de las mujeres tecnólogas a las TIC, sus usos y prácticas se muestran muy diversas, destacando una aplicación muy heterogénea de las TIC. Las mujeres programan desde webs hasta artefactos mecánicos, realizan instalaciones artísticas, manejan bases de datos y administran servidores, componen música electrónica, navegan por Internet, enseñan utilizando las TIC, cosen y lavan ropa con maquinaria doméstica, analizan procesos informáticos, ayudan a los/as usuarios/as de aplicaciones informáticas, producen audiovisuales, graban eventos con la cámara, son realizadoras de e-radio, documentan y desarrollan manuales, escriben epoesía y un largo etcétera, quizás lo que menos se encuentra es el mantenimiento de equipos informáticos, así como temas de seguridad informática. Así lo muestra este testimonio de una profesora de matemáticas e informática: "Tengo mi web, la de mi empresa, escribo cursos directamente en Internet, corrijo los trabajos de mis alumnos telemáticos..."

Aproximadamente la mitad de las mujeres tecnólogas además de usuarias avanzadas son también desarrolladoras y creadoras en algún campo tecnológico específico. Aunque muchas de estas mujeres han recibido su formación reglada en campos diferentes de los tradicionalmente considerados TIC, como la informática o las telecomunicaciones, ellas interseccionan y transdisciplinarizan sus conocimientos y prácticas con las tecnologías. En relación a ello, cabe decir que las mujeres están ocupadas en profesiones y sectores muy variados, y puede que estos constituyan una muestra de los diferentes sectores donde se realiza un uso más intensivo de las TIC. Así, aparte de la informática, encontramos a muchas mujeres ocupadas en el sector de la educación, el mundo artístico, los medios de comunicación y la administración pública. Además, estas no sólo utilizan las TIC para trabajar, sino que utilizan las TIC en su vida cotidiana, para comunicarse, para administrar o agilizar sus tareas domésticas o familiares o para sus propios hobbies y momentos de ocio.

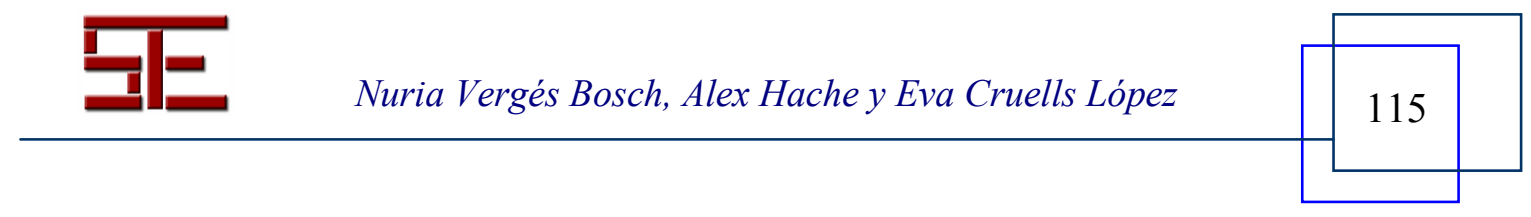




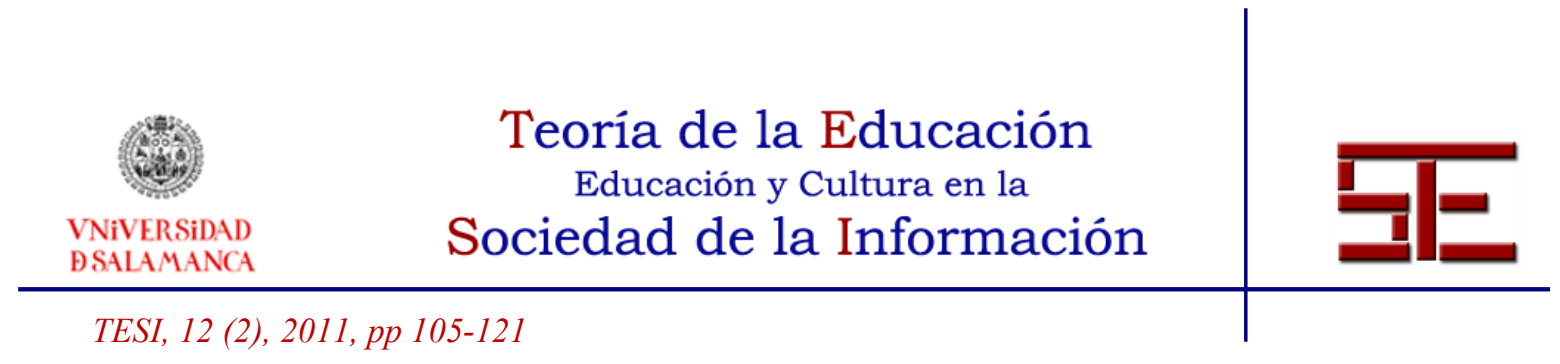

Al contrario de la imagen estereotipada de las personas en las TIC, cuanto más avanzado es el conocimiento y práctica tecnológica de estas mujeres más trabajo en equipo realizan. De hecho podríamos decir que la práctica tecnológica implica una soledad relativa. Esto es, con momentos de aprendizaje o profundización en soledad, pero, a su vez, conectados o compartidos con otras personas en algún momento determinado o como parte del proceso de creación o desarrollo de cualquier nueva aplicación, programa o código. En este sentido Internet juega un papel determinante no sólo como repositorio de documentación, sino como espacio de comunicación e interacción y aprendizaje continuado. Así lo muestra la experiencia de una ingeniera topógrafa: "Intercambio información geomática con otros departamentos de trabajo. En la vida privada soy asidua de foros de Internet como fuente de información $\mathrm{y}$ conocimiento".

Aunque el Hardware que utilizan suele ser de tipo privativo, muy condicionado por la oferta, los usos de software se diversifican mucho más, en cuanto a aplicaciones, pero también en cuanto a sistemas operativos. Así, las mujeres utilizan sobre todo el sistema operativo Windows y en menor medida Gnu/Linux o Mac. Pero cabe decir que prácticamente la mitad de ellas utilizan algún programa de software libre paralelamente a programas privativos. Además, destaca su interés por conocer y difundir lenguajes y aplicaciones libres, también como fórmula de transformación de género. Este hecho es interesante porque contrarresta ciertos estereotipos que consideran que las mujeres no se interesan por el software libre y sus potencialidades transformadoras.

El uso que realizan de las TIC es muy intenso en cuanto a tiempos. Dedican muchas horas de trabajo y personales a su interacción con las tecnologías. Esto les genera multitud de beneficios laborales, personales y a nivel de adquisición de conocimientos, pero, a su vez, problemas de salud. Sus usos son muy intensos a la vez que variados y simultáneos. Cuando son madres o tienen personas a su cargo, el multitasking se intensifica y no siempre resulta fácilmente conciliable su vida laboral y personal con la familiar, implicando una reorganización de sus tiempos y usos tecnológicos.

\subsection{Críticas, sueños y deseos expresados...}

Las mujeres expresan sus deseos de mejora respecto a su práctica tecnológica específica, sobre todo, mencionando la voluntad de incrementar sus conocimientos y extender sus usos tecnológicos. En este sentido quieren, por orden de importancia, mejorar sus conocimientos y habilidades web, en lenguajes y programación, en el uso

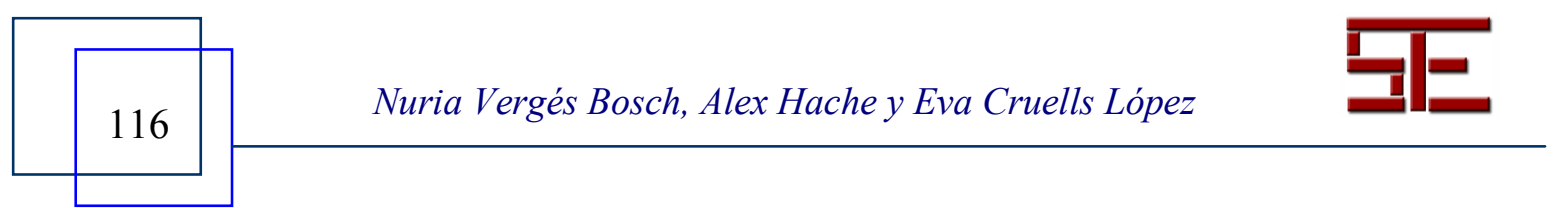




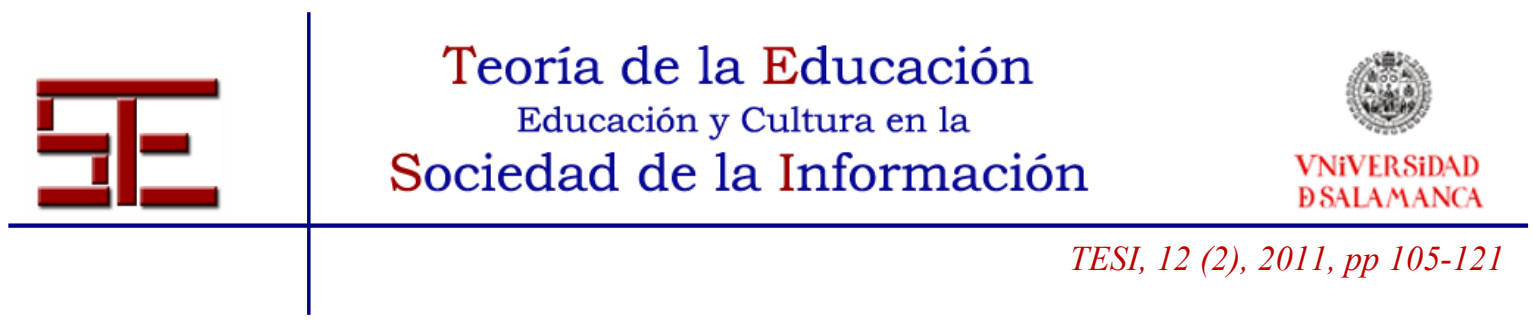

de programas y aplicaciones específicas y en el uso del audiovisual y las tecnologías interactivas, el diseño o las herramientas de gestión de la información. Entre estas cosas, una vez más, encontramos tareas y habilidades muy relacionadas con Internet, como mejorar los conocimientos sobre la creación web, mejorar la transmisión de contenidos y conocimientos por Internet, o mejorar su agilidad y gestión de la información en la navegación, etc. Así lo expresaba una profesora de secundaria: "Más dominio de las herramientas de creación de vídeo, imagen y web. Iniciación a la radio por Internet y participar en proyectos conjuntos que se difundan por Internet”.

Destaca su interés por conocer y difundir lenguajes y aplicaciones libres. Este hecho es interesante porqué contrarresta ciertos estereotipos que consideran que las mujeres no se interesan por el software libre. Así lo manifiesta una artista digital: "Por eso el software libre. En francés sistema operativo se llama système d'exploitation, entonces ser dueñas de tu sistema de explotación es lo mínimo, y cambiarlo es lo mínimo de lo mínimo. También la idea de compartir e intercambiar juntas. Aquí no hay copias, todo el mundo tiene el original, esa posibilidad de compartir al igual las cosas es importante".

Además de incrementar sus conocimientos quieren empoderarse y mejorar sus actitudes en relación a la tecnología, incrementando su autonomía, seguridad o su capacidad de toma de riesgo. Además quieren desarrollar más habilidades beneficiosas para ellas y para el conjunto de la sociedad, como la transmisión del conocimiento adquirido o mejorar la distribución y organización de los tiempos. Pero también quieren hacerlo de una forma mejor y en mejores condiciones que también se relaciona con los contenidos de Internet y el acceso a ellos. Esto es, de una manera que facilite la circulación libre y accesible del conocimiento, con más seguridad y autonomía y de forma más económica y sostenible. Así lo expresaba una informática y estadística: "En el acceso. Las compañías de telefonía/Internet deberían de estar más controladas y dar un mejor servicio en España, como pasa fuera. Es muy caro".

A su vez, expresan los peligros de una evolución tecnológica insostenible o que responda a intereses específicos o comerciales y proponen formas de actuación para transformar la práctica tecnológica actual y facilitar la mejora de la sociedad en su conjunto desde prácticas más colaborativas, sostenibles y donde la persona y el beneficio para la sociedad en su conjunto recuperen su centralidad. Así lo expresa una mujer investigadora: "Una humanidad más respetuosa con la diferencia y con el entorno que la rodea del cual depende. Menos consumo por consumo, menos materialismo e individualismo, más responsabilidad social, más consciencia y más participación".

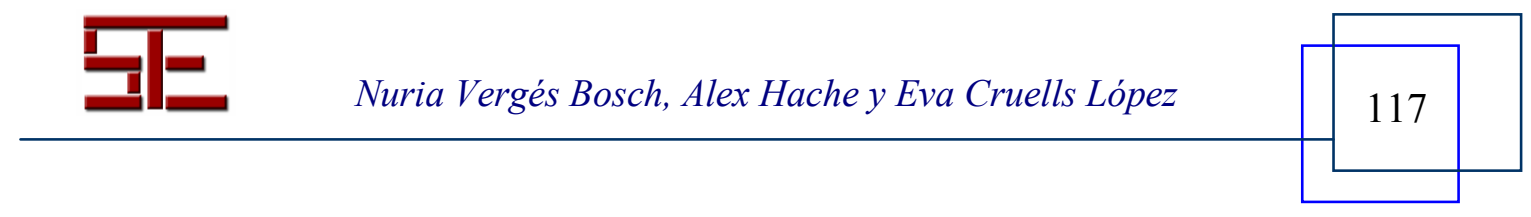




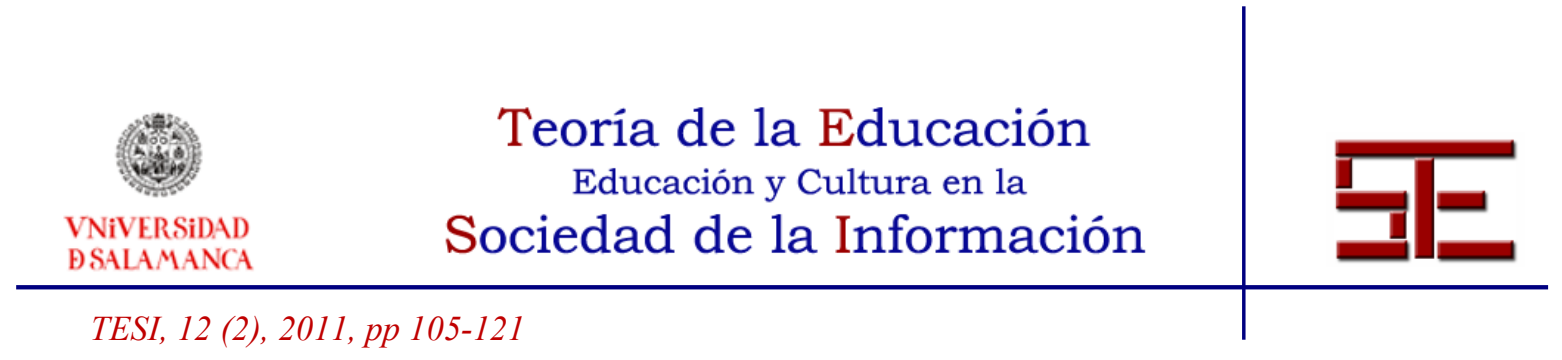

Finalmente, las tecnólogas cuestionan la realidad en la que viven en cuanto a la persistencia de desigualdades sociales, expresando la necesidad de ampliar el acceso a los recursos económicos y a los recursos formativos en materia tecnológica y en mejorar las condiciones laborales. Pero también critican su realidad en la cuestión de género. Aquí las mujeres tecnólogas muestran los principales retos que las mujeres tienen delante en relación a las tecnologías como la necesidad de visibilizar su presencia y contribuciones, la conciliación de la vida laboral y familiar, la mejora en la distribución y realización de tareas domésticas y el fin de la discriminación de género y, sobre todo, la necesidad de romper con los estereotipos de género que las señalan como extrañas o desubicadas tal y como lo exponía una estudiante de Ingeniera Industrial: "Que la gente no te mire extrañada cuando comentas que eres una chica estudiante de electrónica, aficionada a la informática...".

\section{DISCUSIÓN/ CONCLUSIONES}

Las mujeres tecnólogas han accedido y utilizan las tecnologías de formas muy diversas y heterogéneas. Además presentan, sobre todo, trayectorias de inclusión poco lineares. Como se ha visto, Internet juega un papel determinante como elemento de acceso, inmersión y uso tecnológico para las mujeres.

Las mujeres tecnólogas que componen nuestra muestra son mujeres relativamente jóvenes, de grandes ciudades, con estudios universitarios, con trabajos remunerados y una posición social media-alta, aunque económicamente media-baja. Sobre todo, son solteras, tienen pareja, tiempo libre y normalmente no tienen personas a su cargo. Esto implica que en la elaboración de estrategias de inclusión además del género cabe tener en cuenta las desigualdades por lugar de origen, clase y edad, pues las mujeres mayores, de zonas rurales y sin estudios o con pocos recursos económicos pueden estar doblemente excluidas de lo tecnológico.

El resultado del análisis de sus experiencias y opiniones implica considerar las trayectorias poco o nada lineares para facilitar y comprender el acceso y la inmersión de las mujeres en las TIC, pero también las motivaciones entusiastas de las mujeres en relación a las TIC y las vías de aprendizaje informales donde Internet juega un papel determinante. El vacío existente entre su primer contacto y su inmersión tecnológica implica la necesidad de incidir en las edades escolares, pero también la importancia de considerar las trayectorias poco lineares de acceso e inmersión tecnológica. Lo que las

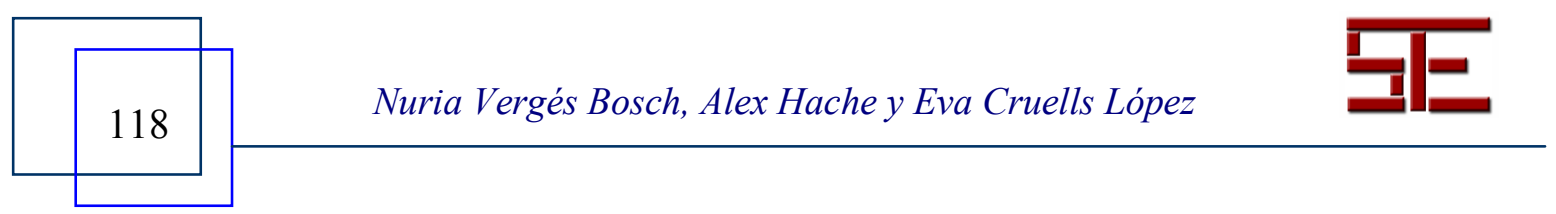




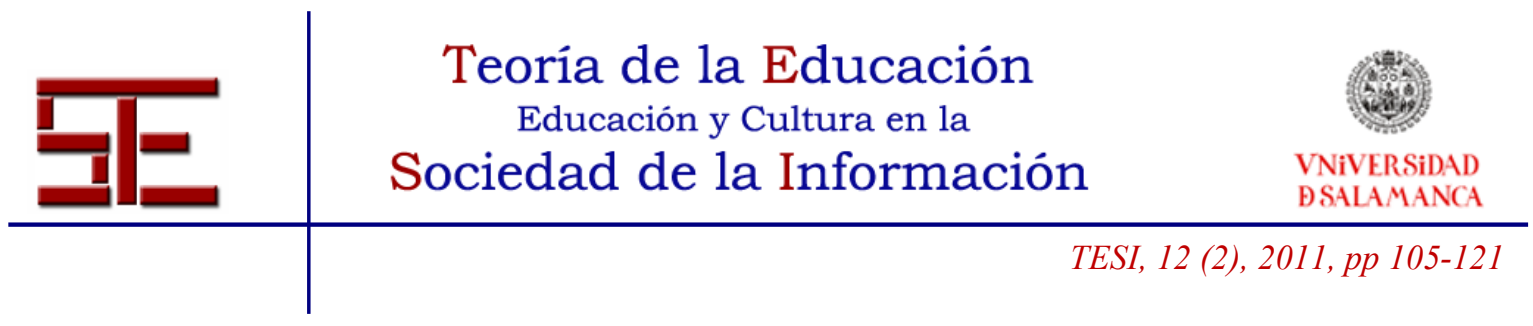

ha motivado personalmente a acceder a las tecnologías ha sido la curiosidad, o la pasión por lo tecnológico, pero también las necesidades formativas o laborales que se han ido encontrando. De este modo compartimos la opinión que las motivaciones entusiastas de las mujeres deben ser consideradas y potenciadas para fomentar el acceso a las TIC de muchas otras más. Ellas consideran que su acceso ha sido posible, sobre todo, gracias a la formación y el trabajo. Pero también, gracias a su capacidad económica y acceso a los equipamientos, su entorno afectivo y social ya relacionado o motivado por las tecnologías, la existencia de Internet y sus posibilidades comunicativas y como repositorio de información, además de otros factores como tener tiempo o el contexto actual que las hace necesarias. Finalmente, las tecnólogas acceden al aprendizaje tecnológico por vías informales en ocasiones de forma simultánea a la educación reglada. Por ello las posibilidades de aprendizaje informal, que en gran medida se relacionan con Internet, deben ser consideradas en el análisis de las experiencias de las mujeres y en el planteamiento de la inclusión e inmersión de las mujeres en las TIC.

Además corroboramos la heterogeneidad y fluidez de la práctica y ámbitos tecnológicos donde se encuentran las mujeres, allí, una vez más, Internet resulta transversal y significante. Además el análisis también muestra el interés de las mujeres por las herramientas libres, la elevada sociabilidad presente en el mundo tecnológico, pero también la intensidad en el trabajo sobre todo en cuanto a tiempos y la persistencia de la discriminación de género. Así pues, las mujeres en las TIC también se han mostrado críticas con el desarrollo tecnológico actual y han expresado sus necesidades y deseos de mejora, tanto de forma individual como colectiva. Prácticamente la totalidad de las mujeres encuestadas quieren saber más y hacer más cosas con las tecnologías. Pero también quieren hacerlo de una forma mejor y en mejores condiciones. Esto es, de una manera más sostenible, que facilite la circulación libre y accesible del conocimiento, con más seguridad y autonomía y con mejores condiciones laborales y distribuciones del tiempo y, sobre todo, de una manera que facilite un acceso igualitario y justo a las tecnologías y que acabe con las discriminaciones y dicotomías de género existentes.

\section{Agradecimientos}

Queremos agradecer especialmente a los cientos de mujeres que han compartido sus experiencias y opiniones con nosotras. Este trabajo se basa en dos proyectos de investigación. El primero financiado por el Institut Català de les Dones, Generalitat de Catalunya, con el título "Accés i usos de les tecnologies per part de les dones a

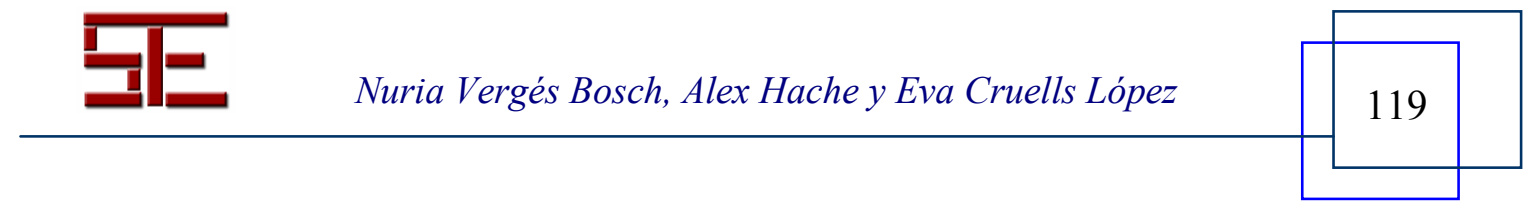




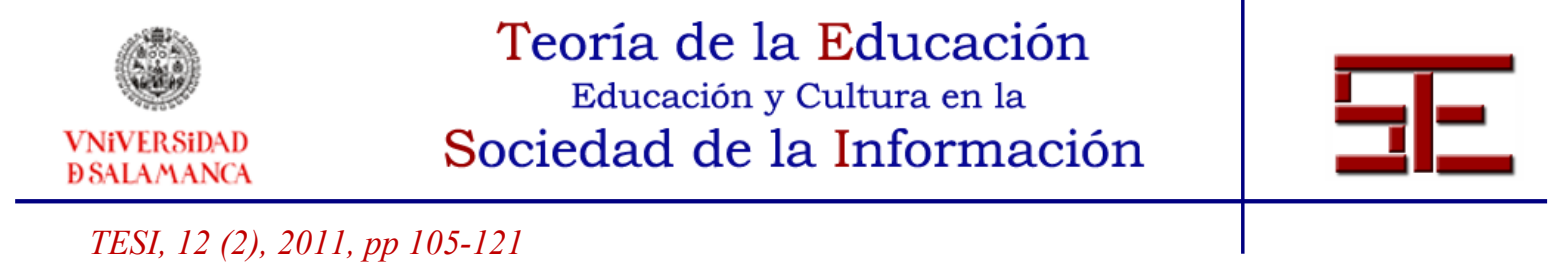

Catalunya" (PF-36/06). El segundo financiado por el Plan Avanza, Ministerio de Industria, Comercio y Turismo, con el título "Investigación sobre el acceso y uso técnico de las tecnologías por parte de las mujeres en el Estado Español" (PAV-0100002006-075).

\section{BIBLIOGRAFÍA}

Cohoon J. G. Aspray, W. (2006). Ed. Women and Information Technology: Research on Under-Representation. Masachussets: MIT Press.

Faulkner, Wendy \& Lie, M. (2007). Gender in the Information Society: Strategies of Inclusion. Gender Technology and Development. 11 (2), 157-177.

Flanagan, M. \& Booth, A. (2002). Reload. Rethinking women + cyberulture. Massachussetts: MIT.

Haraway, D. (1991). Simians, Cyborgs and Women. New York: Routledge.

Hawthorne, S. \& Klein, R. (1999). Cyberfeminism: Connectivity, Critique + Creativity. Australia: Spinifex.

Lagesen, V. (2007). The Strength of Numbers: Strategies to Include Women into Computer Science. Social Studies of Science, 37, 67.

Lagesen, V. (2008). A Cyberfeminist Utopia? Perceptions of Gender and Computer Science among Malysian Women Computer Science Students and Faculty. Science, Technology \& Human Values, 33 (1), 5-27.

Landström, C. (2007). Queering feminist technology studies. Feminist Theory, 8 (7), 726.

Lerman, N . E., Oldenziel, R. \& Mohun, A P. (2003). Introduction: Interrogating Boundaries. En Lerman et al., Gender and Technology. A reader. The Johns Hopkins University Press.

Margolis, J. \& Fisher, A. (2001). Unlocking the clubhouse. Women in Computing. London: The MIT Press.

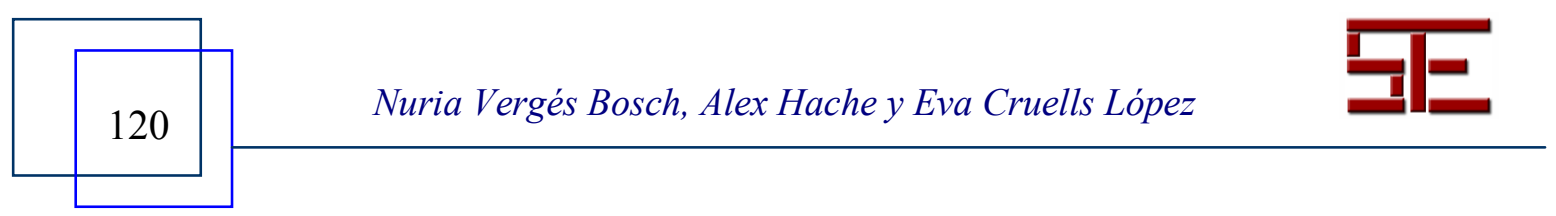




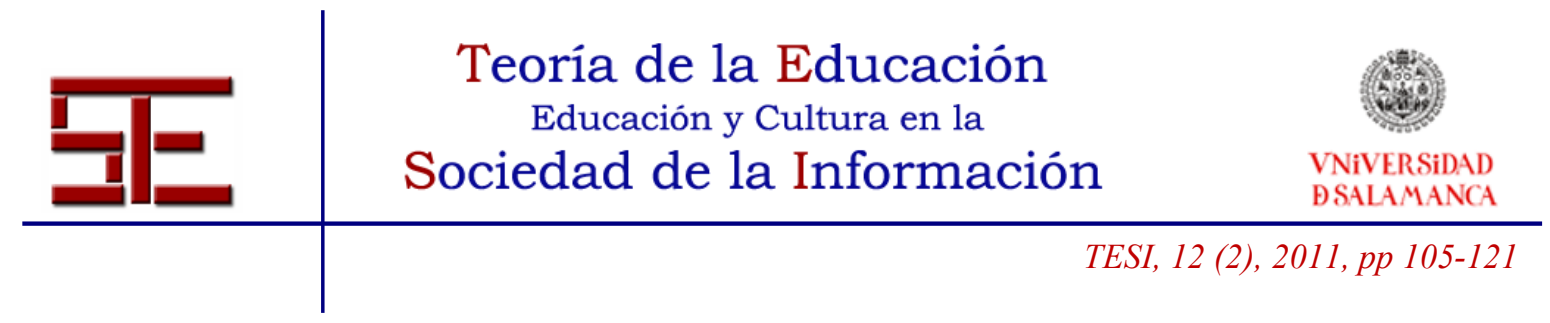

Núñez, S. \& Garcia, A. (2009). New technologies and new spaces for Relation. Spanish Feminist Praxis Online. European Journal of Women's Studies, 16, 249.

Plant, S. (1997). Zeros + Ones. Digital women + the new technoculture. New Cork: Doubleday.

Sorensen, K. H. (2002). Love, Duty and the S-curve: An Overview of Some Current Literature on Gender and ICT. Sigis. Deliverable Number, D02_Part 1, 1-36.

Sveningson, M. \& Sunden, J. (2007). Cyberfeminism in Northern Lights: Digital Media and Gender in a Nordic Context. Cambridge: Cambridge Scholars Publishing.

Vergés, N., Cruells E. \& Hache, A (2010). Cyberfeminismo de Investigacción. En Zafra. Ensayos sobre género y ciberespacio. Madrid: Briseño editores.

Wajcman, J. (2004). Technofeminism. Cambridge: Polity Press.

Para citar el presente artículo puede utilizar la siguiente referencia:

Vergés Bosch, N., Hache, A. y Cruells Lopez, E. (2011). Indagando en la relevancia de Internet en el acceso, uso y deseos de las TIC por parte de las mujeres en las TIC, en Barrios Vicente, I. M. (Coord.) Mujeres y la sociedad de la Información. Revista Teoría de la Educación: Educación y Cultura en la Sociedad de la Información. Vol. 12, $\mathrm{n}^{\circ}$ 2. Universidad de Salamanca, pp. 105-121 [Fecha de consulta: dd/mm/aaaa]. http://campus.usal.es/ revistas_trabajo/index.php/revistatesi/article/view/8275/8279

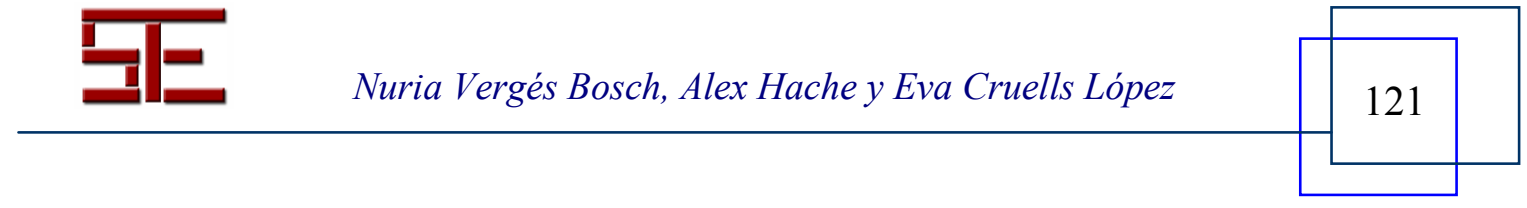

